PROCEEDINGS OF THE

AMERICAN MATHEMATICAL SOCIETY

Volume 135, Number 7, July 2007, Pages 2011-2018

S 0002-9939(07)08703-5

Article electronically published on March 2, 2007

\title{
PROOF OF THE BARKER ARRAY CONJECTURE
}

\author{
JAMES A. DAVIS, JONATHAN JEDWAB, AND KEN W. SMITH
}

(Communicated by John R. Stembridge)

\begin{abstract}
Using only elementary methods, we prove Alquaddoomi and Scholtz's conjecture of 1989, that no $s \times t$ Barker array having $s, t>1$ exists except when $s=t=2$.
\end{abstract}

\section{INTRODUCTION}

Binary sequences and arrays whose out-of-phase aperiodic autocorrelations are collectively small are particularly useful in digital communication systems, especially synchronisation and radar. The search for such sequences and arrays dates from the 1950s [2, [16] and continues to the present day [7, 9], 13, 14. We define an $s \times t$ array to be a two-dimensional array $\left(a_{i j}\right)$ of complex-valued elements satisfying

$$
a_{i j}=0 \text { unless } 0 \leq i<s \text { and } 0 \leq j<t .
$$

The array is binary if all nonzero elements $a_{i j}$ take values in $\{1,-1\}$. The aperiodic autocorrelation function of an $s \times t$ array $A=\left(a_{i j}\right)$ is given by

$$
C_{A}(u, v)=\sum_{i} \sum_{j} a_{i j} \overline{a_{i+u, j+v}} \text { for integer } u, v \text { satisfying }|u|<s \text { and }|v|<t .
$$

We refer to an $s \times 1$ array as a sequence of length $s$, abbreviating the array $\left(a_{i 0}\right)$ to $\left(a_{i}\right)$ and its aperiodic autocorrelation function $C_{A}(u, 0)$ to $C_{A}(u)$.

Alquaddoomi and Scholtz [1] defined an $s \times t$ Barker array to be an $s \times t$ binary array $A$ for which

$$
\left|C_{A}(u, v)\right| \leq 1 \text { for all }(u, v) \neq(0,0)
$$

This generalises the notion of a Barker sequence from one dimension (the case $s=1$ or $t=1$ ) to two dimensions; see [10] and [1] for recent nonexistence results for Barker sequences. The $2 \times 2$ array $\left[{ }_{+}^{+}+{ }_{+}^{+}\right]$is a Barker array, but it is conjectured that there are no other sizes for a (truly two-dimensional) Barker array.

Conjecture 1.1 (Alquaddoomi and Scholtz [1]). If an $s \times t$ Barker array exists for $s, t>1$, then $s=t=2$.

Received by the editors October 25, 2005 and, in revised form, March 10, 2006.

2000 Mathematics Subject Classification. Primary 05B10; Secondary 94 A99.

Key words and phrases. Barker array, difference set, relative difference set, perfect array, quasiperfect array.

The first author was supported by grant \# MDA904-03-1-0032 (NSA).

The second author was supported by grant \# 31-611394 (NSERC Canada).

The third author received sabbatical support from Central Michigan University and gracious hospitality from the University of Richmond.

(C)2007 American Mathematical Society Reverts to public domain 28 years from publication 
In this paper we prove Conjecture 1.1 using only elementary methods. We include short proofs of key auxiliary results obtained elsewhere, in order to make the paper self-contained. Theorem 1.2 summarises the previous state of knowledge regarding Conjecture 1.1.

Theorem 1.2 (Jedwab 6], Jedwab, Lloyd and Mowbray [8]). Let $A$ be an $s \times t$ Barker array with $s, t>1$. Then

Case 1. $s, t$ even: $s=t$. If $t>2$, then $t \equiv 0(\bmod 4)$ and $t \geq 12$.

Case 2. $s$ even, $t>1$ odd: $s>t . s=4 S^{2}$ and $t=T^{2}$ for integers $S, T$. There exists a Barker sequence of length $s$.

Case 3. $s, t>1$ odd: st $\geq 3^{11}$. Write $t=\prod_{j} p_{j}^{\alpha_{j}}$, where the $\left\{p_{j}\right\}$ are distinct primes and $\alpha_{j} \geq 1$ for all $j$. Then $\alpha_{j} \geq 2$ for all $j$ and $\alpha_{k}>2$ for some $k$. If st $\equiv 1(\bmod 4)$, then $p_{j} \equiv 1(\bmod 4)$ for all $j$.

Following [1], define the following function for an $s \times t$ array $A=\left(a_{i j}\right)$ :

$$
P_{A}(u, v)=C_{A}(u, v)+C_{A}(u, v-t) \text { for }-s<u<s \text { and } 0 \leq v<t .
$$

Any expression involving $P_{A}(u, v)$ or $C_{A}(u, v)$ will implicitly refer only to values of $(u, v)$ for which the function is defined. In terms of the array elements $a_{i j}$ we have

$$
P_{A}(u, v)=\sum_{i} \sum_{j=0}^{t-1} a_{i j} \overline{a_{i+u,(j+v) \bmod t}} .
$$

Alquaddoomi and Scholtz [1] established Lemma 1.3 for binary arrays, and then used it to prove Proposition 1.4 for Barker arrays. This generalised the approach taken by Tuyrn and Storer in their classical paper [15] on the one-dimensional (sequence) case.

Lemma 1.3 (Alquaddoomi and Scholtz [1]). Let $A$ be an $s \times t$ binary array. Then

$$
P_{A}(u, v) \equiv P_{A}\left(u, v^{\prime}\right) \quad(\bmod 4) \text { for all }\left(u, v, v^{\prime}\right) \text {. }
$$

Proof. Let $u, v, v^{\prime}$ satisfy $-s<u<s$ and $0 \leq v, v^{\prime}<t$. From (1.2), $P_{A}(u, v)$ is the sum of $(s-|u|) t$ nonzero terms, of which exactly $\left[(s-|u|) t-P_{A}(u, v)\right] / 2$ are -1 and $\left[(s-|u|) t+P_{A}(u, v)\right] / 2$ are +1 . But from (1.2), the product of these nonzero terms is independent of $v$. Therefore

$$
(-1)^{\left[(s-|u|) t-P_{A}(u, v)\right] / 2}
$$

is independent of $v$, which implies $P_{A}(u, v) \equiv P_{A}\left(u, v^{\prime}\right)(\bmod 4)$.

Proposition 1.4 (Alquaddoomi and Scholtz [1]). Let $A$ be an $s \times t$ Barker array with st $>2$. Then

Case 1. $s, t$ even:

$$
P_{A}(u, v)=0 \text { for }(u, v) \neq(0,0) .
$$

Case 2. $s$ even and $t$ odd:

$$
\begin{aligned}
P_{A^{T}}(v, u) & =0 \text { for }(u, v) \neq(0,0), \\
P_{A}(u, v) & = \begin{cases}0 & \text { for } u \text { even and }(u, v) \neq(0,0), \\
k(u) & \text { for } u \text { odd },\end{cases}
\end{aligned}
$$

where $k(u)=1$ or -1 . 
Case 3. $s, t$ odd:

$$
P_{A}(u, v)= \begin{cases}k & \text { for } u \text { even and }(u, v) \neq(0,0), \\ 0 & \text { for } u \text { odd },\end{cases}
$$

where $k=1$ or -1 .

Proof. For all $u, v$ satisfying $|u|<s$ and $|v|<t, C_{A}(u, v)$ is the sum of $(s-|u|)$ $\times(t-|v|)$ nonzero terms, each of which is \pm 1 . Therefore $C_{A}(u, v) \equiv(s+u)(t+v)$ $(\bmod 2)$. The Barker array property then implies

$$
C_{A}(u, v)= \pm(((s+u)(t+v)) \bmod 2) \text { for }(u, v) \neq(0,0) .
$$

Case 1. s, $t$ even: From (1.3) we have

$$
C_{A}(u, v)=0 \text { for } u \text { or } v \text { even and }(u, v) \neq(0,0) .
$$

Then by (1.1),

$$
P_{A}(u, v)=0 \text { for } u \text { or } v \text { even and }(u, v) \neq(0,0) .
$$

Lemma 1.3 then implies that

$$
P_{A}(u, v)=0 \text { for }(u, v) \neq(0,0) .
$$

Case 2. s even, $t$ odd: From (1.3) we have

$$
C_{A}(u, v)= \pm((u(1+v)) \bmod 2) \text { for }(u, v) \neq(0,0) .
$$

It follows from (1.1) that

$$
P_{A}(u, v)= \begin{cases}0 & \text { for } u \text { even and }(u, v) \neq(0,0) \\ \pm 1 & \text { for } u \text { odd }\end{cases}
$$

Lemma 1.3 then implies that

$$
P_{A}(u, v)= \begin{cases}0 & \text { for } u \text { even and }(u, v) \neq(0,0), \\ k(u) & \text { for } u \text { odd }\end{cases}
$$

where $k(u)=1$ or -1 , as required.

We next consider the function

From (1.4),

$$
P_{A^{T}}(v, u)=C_{A}(u, v)+C_{A}(u-s, v) .
$$

$$
P_{A^{T}}(v, u)=0 \text { for } u \text { even and }(u, v) \neq(0,0) .
$$

Lemma 1.3 applied to $A^{T}$ states that

$$
P_{A^{T}}(v, u) \equiv P_{A^{T}}\left(v, u^{\prime}\right) \quad(\bmod 4) \text { for all }\left(u, u^{\prime}, v\right),
$$

giving

$P_{A^{T}}(v, u)=0$ for $(u, v) \neq(0,0)$, except when $s=2$ and $(u, v)=(1,0)$

(since, when $s=2$ and $v=0$, there is no value of $u$ satisfying the conditions of (1.7) ).

To complete the proof of Case 2, we now derive a contradiction for the case $s=2$, so that (1.8) holds without exception. By assumption $s t>2$ and $s=2$, so $t>1$ and we can choose an even value of $v$ satisfying $0<v<t$. From (1.5),

$$
k(1)=P_{A}(1, v)=P_{A}(1, t-v),
$$


and so from (1.1) and (1.4),

$$
\pm 1=C_{A}(1, v)=C_{A}(1,-v) .
$$

But by (1.8), $P_{A^{T}}(v, 1)=0$, and so from (1.6) we get

$$
\begin{aligned}
0 & =C_{A}(1, v)+C_{A}(-1, v) \\
& =C_{A}(1, v)+C_{A}(1,-v)
\end{aligned}
$$

since $C_{A}(u, v)=C_{A}(-u,-v)$ for all $u, v$. This contradicts (1.9).

Case 3. $s, t$ odd: From (1.3) we have

$$
C_{A}(u, v)= \pm(((1+u)(1+v)) \bmod 2) \text { for }(u, v) \neq(0,0) .
$$

Then by (1.1),

$$
P_{A}(u, v)= \begin{cases} \pm 1 & \text { for } u \text { even and }(u, v) \neq(0,0) \\ 0 & \text { for } u \text { odd }\end{cases}
$$

Lemma 1.3 then implies that

$$
P_{A}(u, v)= \begin{cases}k(u) & \text { for } u \text { even and }(u, v) \neq(0,0), \\ 0 & \text { for } u \text { odd }\end{cases}
$$

where $k(u)=1$ or -1 . By symmetry in $s$ and $t$ we also obtain

$$
P_{A^{T}}(v, u)= \begin{cases}k^{\prime}(v) & \text { for } v \text { even and }(u, v) \neq(0,0), \\ 0 & \text { for } v \text { odd }\end{cases}
$$

where $k^{\prime}(v)=1$ or -1 . But, for $u, v$ even and $(u, v) \neq(0,0)$, by (1.3) the single nonzero contribution to $P_{A}(u, v)=C_{A}(u, v)+C_{A}(u, v-t)$ and to $P_{A^{T}}(v, u)=C_{A}(u, v)+C_{A}(u-s, v)$ is the same term $C(u, v)$, and so $k(u)=k^{\prime}(v)=k$.

Proposition 1.4 is implied by Theorem 2 and (21)-(23) of [1]. Lemma 3.5 of $[6$ shows that an $s \times t$ binary array $A$ having $P_{A}(u, v)=0$ for all $(u, v) \neq(0,0)$ is equivalent to $A$ being simultaneously a perfect binary array and a "quasiperfect" binary array. This in turn is equivalent to the -1 elements of $A$ corresponding to a $\left(4 N^{2}, 2 N^{2}-N, N^{2}-N\right.$ )-difference set in $\mathbb{Z}_{s} \times \mathbb{Z}_{t}$, where $s t=4 N^{2}$ (see [4, for example), and the -1 elements of $\left[\begin{array}{r}A \\ -A\end{array}\right]$ corresponding to an $(s t, 2, s t, s t / 2)$ relative difference set in $\mathbb{Z}_{2 s} \times \mathbb{Z}_{t}=\langle x\rangle \times\langle y\rangle$, where $x^{2 s}=y^{t}=1$, relative to $\left\langle x^{s}\right\rangle$ (see [17]). See [3] or [12] for a background on difference sets and relative difference sets.

\section{Proof of the conjecture}

We begin with two lemmas.

Lemma 2.1. Let $A=\left(a_{i j}\right)$ be an $s \times t$ binary array and let $\zeta$ be a (not necessarily primitive) $t^{\text {th }}$ root of unity. Let $X=\left(x_{i}\right)$ be the complex-valued sequence of length $s$ given by

$$
x_{i}=\sum_{j} a_{i j} \zeta^{j} .
$$

Then

$$
C_{X}(u)=\sum_{v=0}^{t-1} P_{A}(u, v) \zeta^{-v} \text { for all } u
$$


Proof. From (1.2), for all $u$,

$$
\begin{aligned}
\sum_{v=0}^{t-1} P_{A}(u, v) \zeta^{-v} & =\sum_{v=0}^{t-1} \sum_{i} \sum_{j} a_{i j} \overline{a_{i+u,(j+v) \bmod t}} \zeta^{-v} \\
& =\sum_{i} \sum_{j} a_{i j} \sum_{k=0}^{t-1} \overline{a_{i+u, k}} \zeta^{j-k},
\end{aligned}
$$

writing $k=(j+v) \bmod t$ and using $\zeta^{t}=1$. Hence, for all $u$,

$$
\begin{aligned}
\sum_{v=0}^{t-1} P_{A}(u, v) \zeta^{-v} & =\sum_{i} \sum_{j} a_{i j} \zeta^{j} \overline{\sum_{k} a_{i+u, k} \zeta^{k}} \\
& =\sum_{i} x_{i} \overline{x_{i+u}} \\
& =C_{X}(u),
\end{aligned}
$$

as required.

Lemma 2.2. Let $X=\left(x_{i}\right)$ be a complex-valued sequence of length s for which

$$
C_{X}(u)=0 \text { for } u \neq 0 .
$$

Then, for some I satisfying $0 \leq I<s$,

$$
\left|x_{i}\right|^{2}= \begin{cases}0 & \text { for } i \neq I \\ C_{X}(0) & \text { for } i=I\end{cases}
$$

Proof. By the definition of aperiodic autocorrelation, we are given that

$$
\sum_{i} x_{i} \overline{x_{i+u}}=0 \text { for } 0<u<s .
$$

We prove by induction on $s$ that, for some $I$ satisfying $0 \leq I<s$,

$$
\left|x_{i}\right|^{2}=0 \text { for } i \neq I \text {. }
$$

The case $s=1$ is immediate (take $I=0$ ). Assume case $s-1$ to be true. Put $u=s-1$ in (2.2) to give $x_{0} \overline{x_{s-1}}=0$. This implies, without loss of generality, that $x_{s-1}=0$. Then from (2.2) we have

$$
\sum_{i=0}^{s-u-2} x_{i} \overline{x_{i+u}}=0 \text { for } 0<u<s-1 .
$$

By the inductive hypothesis it follows that, for some $I$ satisfying $0 \leq I<s-1$, $\left|x_{i}\right|^{2}=0$ for $i \neq I$. Combining this with $x_{s-1}=0$ gives the case $s$, completing the induction.

Furthermore, by the definition of aperiodic autocorrelation, $C_{X}(0)=\sum_{i}\left|x_{i}\right|^{2}$, and so $C_{X}(0)=\left|x_{I}\right|^{2}$, as required.

The case $\zeta=1$ of Lemma 2.1] was used as a starting point in [5], 6] and [8] to derive equations in the row sums $\sum_{j} a_{i j}$ of an $s \times t$ Barker array from Proposition 1.4, eventually leading to Theorem 1.2. We will now use the case where $\zeta$ is a primitive $t^{\text {th }}$ root of unity to prove Conjecture 1.1 .

Theorem 2.3. If an $s \times t$ Barker array $A=\left(a_{i j}\right)$ exists for $s, t>1$, then $s=t=2$. 
Proof. Let $\zeta$ be a primitive $t^{\text {th }}$ root of unity and define $X=\left(x_{i}\right)$ as in (2.1). We will show that the case $s, t$ even forces the result $s=t=2$, whereas the case $s$ even, $t$ odd and the case $s, t$ odd both result in a contradiction. These three cases are exhaustive, because the transpose of a Barker array is also a Barker array.

Case 1. s, $t$ even: Proposition 1.4 and Lemma 2.1 together give

$$
C_{X}(u)= \begin{cases}0 & \text { for } u \neq 0, \\ s t & \text { for } u=0,\end{cases}
$$

using $P_{A}(0,0)=C(0,0)=s t$. Then by Lemma 2.2 there is some $I$ satisfying $0 \leq I<s$ for which

$$
\left|x_{I}\right|^{2}=s t
$$

But by (2.1),

$$
\begin{aligned}
\left|x_{I}\right|^{2} & =\left|\sum_{j=0}^{t-1} a_{I j} \zeta^{j}\right|^{2} \\
& \leq\left(\sum_{j=0}^{t-1}\left|a_{I j} \zeta^{j}\right|\right)^{2} \\
& =t^{2}
\end{aligned}
$$

It follows from (2.3) that

(2.4) $s \leq t$, with equality $\Leftrightarrow \arg \left(a_{I j} \zeta^{j}\right)$ is constant for all $j$ satisfying $0 \leq j<t$.

Since $s$ is even, by symmetry in $s$ and $t$ (or equivalently by applying the same procedure to $A^{T}$ ) we have $t \leq s$, forcing equality. Therefore $s=t$ and, since $t>1$, by (2.4) we have $t=2$.

Case 2. $s$ even, $t>1$ odd: By Proposition 1.4, the $t \times s$ array $A^{T}$ satisfies

$$
P_{A^{T}}(v, u)=0 \text { for }(u, v) \neq(0,0) .
$$

The argument of Case 1 that led to (2.4), when applied to $A^{T}$, gives $t \leq$ s. Furthermore the expression for $P_{A}$ in Proposition 1.4, together with Lemma 2.1, gives

$$
\begin{aligned}
C_{X}(u) & = \begin{cases}0 & \text { for } u \text { even and } u \neq 0, \\
k(u) \sum_{v=0}^{t-1} \zeta^{-v} & \text { for } u \text { odd } \\
s t & \text { for } u=0\end{cases} \\
& = \begin{cases}0 & \text { for } u \neq 0, \\
s t & \text { for } u=0,\end{cases}
\end{aligned}
$$

since $\zeta^{-1}$ is a primitive $t^{\text {th }}$ root of unity and $t>1$. By Lemma 2.2 we then obtain $s \leq t$, by the same argument as in Case 1 . Since we already have $t \leq s$ this implies $s=t$, which contradicts the assumption that $s$ is even and $t$ is odd. 
Case 3. $s, t>1$ odd: Proposition 1.4 and Lemma 2.1 together give

$$
\begin{aligned}
C_{X}(u) & = \begin{cases}k \sum_{v=0}^{t-1} \zeta^{-v} & \text { for } u \text { even and } u \neq 0, \\
0 & \text { for } u \text { odd } \\
s t+k \sum_{v=1}^{t-1} \zeta^{-v} & \text { for } u=0\end{cases} \\
& = \begin{cases}0 & \text { for } u \neq 0, \\
s t-k & \text { for } u=0,\end{cases}
\end{aligned}
$$

where $k=1$ or -1 . Then by Lemma 2.2 there is some $I$ satisfying $0 \leq I<s$ for which

$$
\left|x_{I}\right|^{2}=s t-k \text {. }
$$

But, as in Case $1,\left|x_{I}\right|^{2} \leq t^{2}$ and so

$$
s t-k \leq t^{2} \text {. }
$$

By symmetry in $s$ and $t$ we then have

$$
s t-k \leq \min \left\{s^{2}, t^{2}\right\} .
$$

Suppose, for a contradiction, that $s \neq t$ and without loss of generality that $s \geq t+1$. Then $s t-k \geq t(t+1)-k>t^{2}$, since $k=1$ or -1 and $t>1$. This contradicts (2.6), and so $s=t$.

Then (2.6) forces $k=1$, and from (2.1) and (2.5) we have

$$
\left|\sum_{j=0}^{t-1} a_{I j} \zeta^{j}\right|^{2}=t^{2}-1 .
$$

Since $t$ is odd, one of the sets $\left\{j: a_{I j}=1\right\}$ and $\left\{j: a_{I j}=-1\right\}$ contains at most $(t-1) / 2$ elements; without loss of generality, suppose it is the former. This implies that

$$
\begin{aligned}
\left|\sum_{j=0}^{t-1} a_{I j} \zeta^{j}\right|^{2} & =\left|\sum_{j=0}^{t-1} a_{I j} \zeta^{j}+\sum_{j=0}^{t-1} \zeta^{j}\right|^{2} \\
& =\left|2 \sum_{j: a_{I j}=1} \zeta^{j}\right|^{2} \\
& \leq 4\left(\sum_{j: a_{I j}=1}\left|\zeta^{j}\right|\right)^{2} \\
& \leq 4\left(\frac{t-1}{2}\right)^{2} \\
& <t^{2}-1,
\end{aligned}
$$

since $t>1$. This contradicts (2.7).

\section{ACKNOWLEDGMENTS}

We are grateful to Denis Dmitriev for supplying the above argument that (2.7) cannot hold for odd $t>1$, which is much neater than our original reasoning. 


\section{REFERENCES}

1. S. Alquaddoomi and R.A. Scholtz, On the nonexistence of Barker arrays and related matters, IEEE Trans. Information Theory 35 (1989), 1048-1057. MR.1023242 (90h:05035)

2. R.H. Barker, Group synchronizing of binary digital systems, Communication Theory (W. Jackson, ed.), Academic Press, New York, 1953, pp. 273-287.

3. T. Beth, D. Jungnickel, and H. Lenz, Design theory, 2nd ed., Cambridge University Press, Cambridge, 1999, Volumes I and II. MR1729456 (2000h:05019) MR1742365 (2000j:05002)

4. Y.K. Chan, M.K. Siu, and P. Tong, Two-dimensional binary arrays with good autocorrelation, Information and Control 42 (1979), 125-130. MR0542326 (80j:94029)

5. J. Jedwab, Nonexistence results for Barker arrays, The Institute of Mathematics and its Applications Conference Series (New Series) No. 33: Cryptography and Coding II (C. Mitchell, ed.), Clarendon Press, Oxford, 1992, pp. 121-126. MR1165734 (93f:11021)

6. _ Barker arrays I: Even number of elements, SIAM J. Discrete Math. 6 (1993), 294308. MR $1215236(94 \mathrm{k}: 05047 \mathrm{a})$

7. J. Jedwab, A survey of the merit factor problem for binary sequences, Sequences and Their Applications - Proceedings of SETA 2004 (T. Helleseth et al., eds.), Lecture Notes in Computer Science, vol. 3486, Springer-Verlag, Berlin, Heidelberg, 2005, pp. 30-55.

8. J. Jedwab, S. Lloyd, and M. Mowbray, Barker arrays II: Odd number of elements, SIAM J. Discrete Math. 6 (1993), 309-328. MR1215237 (94k:05047b)

9. J. Jedwab and K. Yoshida, The peak sidelobe level of families of binary sequences, IEEE Trans. Inform. Theory 52 (2006), 2247-2254.

10. K.H. Leung, S.L. Ma, and B. Schmidt, Nonexistence of abelian difference sets: Lander's conjecture for prime power orders, Trans. Amer. Math. Soc. 356 (2004), 4343-4358. MR2067122 (2005f:05025)

11. K.H. Leung and B. Schmidt, The field descent method, Designs, Codes and Cryptography 36 (2005), 171-188.

12. A. Pott, Finite geometry and character theory, Lecture Notes in Mathematics 1601, SpringerVerlag, Berlin, 1995. MR.1440858 (98j:05032)

13. G.S. Ramakrishna and W.H. Mow, A new search for optimal binary arrays with minimum peak sidelobe levels, Sequences and Their Applications — Proceedings of SETA 2004 (T. Helleseth et al., eds.), Lecture Notes in Computer Science, vol. 3486, Springer-Verlag, Berlin, Heidelberg, 2005 , pp. $355-360$.

14. H.D. Schotten and H.D. Lüke, On the search for low correlated binary sequences, AEU Int. J. of Electronics and Communications 59 (2005), 67-78.

15. R. Turyn and J. Storer, On binary sequences, Proc. Amer. Math. Soc. 12 (1961), 394-399. MR0125026 (23:A2333)

16. R.J. Turyn, Sequences with small correlation, Error Correcting Codes (H.B. Mann, ed.), Wiley, New York, 1968, pp. 195-228. MR0242566 (39:3897)

17. P. Wild, Infinite families of perfect binary arrays, Electron. Lett. 24 (1988), 845-847.

Department of Mathematics and Computer Science, University of Richmond, RichMOND, VIRGINIA 23173

E-mail address: jdavis@richmond.edu

Department of Mathematics, Simon Fraser University, 8888 University Drive, Burnaby, British Columbia, Canada V5A 1S6

E-mail address: jed@sfu.ca

Department of Mathematics, Central Michigan University, Mount Pleasant, MichiGAN 48859

E-mail address: Ken.W.Smith@cmich.edu 